\title{
Prognostic value of serum Uric Acid in-hospital Mortality and Morbidity in Patients with acute Myocardial Infarction
}

MD. DELDAR HOSSAIN ${ }^{1}$, SHEULY FERDOUSI ${ }^{2}$, MD. SAIFUL ISLAM ${ }^{2}$, DEBATOSH PAUL ${ }^{2}$, TTUHIN SULTANA ${ }^{2}$, HARISUL HOQUE ${ }^{3}$, MD QUDDUSUR RAHMAN ${ }^{2}$, SAJAL KRISHNA BANERJEE ${ }^{3}$, AN NASHIMUDDINAHMED ${ }^{2}$

${ }^{1}$ Department of Pathology, Kushtia Medical College, Kushtia, ${ }^{2}$ Department of Laboratory Medicine, Bangabandhu Sheikh Mujib Medical University, Dhaka, ${ }^{3}$ Department of Cardiology, Bangabandhu Sheikh Mujib Medical University, Dhaka. Address of Correspondence: Md. Deldar Hossain, Department of Pathology, Kushtia Medical College, Kushtia

\begin{abstract}
:
Coronary artery disease (CAD) is one of the leading causes of death in the world and also in Bangladesh. This study was carried out to evaluate that Serum uric acid is a prognostic tool in acute myocardial infarction. In this study, 108 AMI cases were enrolled. All the patients were treated and managed identically by conventional standard management protocol. During hospital stay, patients were assessed for any kind of clinical outcomes e.g good recovery, mortality and morbidity (acute LVF, cardiogenic shock, arrhythmia and heart block). Patients with higher serum uric acid (SUA>6.5mg\%, Group-II) were compared with Patients with lower serum uric acid (SUA<6.5mg\%, Group-I). In Group I ( $n=66)$, good recovery, morbidity and mortality were found in 43 (65.2\%), 21 (31.8\%), 2(3.0\%) patients and in Group II (n=42), good recovery, morbidity, mortality were 13 (31\%), 23 (54.8\%) and 6 (14.3\%) patients respectively. In group II patients having high Serum uric acid concentration had low good recovery but high morbidity and mortality compared to group I patients. In group I ( $n=66)$, good outcome and bad outcome was found in 43(65.2\%), and $23(34.8 \%)$ patients and in group II (n=42), those were in $13(31 \%)$ and 29 (69\%) patents. Logistic regression analysis of serum uric acid concentration of study subjects were done considering uric acid as independent variable but outcome as dependent variable. It was shown that the odds ratio of mortality was $5.38(95 \%$ CI $p<0.007)$, odds ratio of morbidity was $4.10(95 \%$ CI $p<0.001)$ and odds ratio of bad outcome was $4.67(95 \%$ CI $p<0.001)$. This findings indicates a patient having high uric acid had 5.38 times increased mortality, 4.10 times increased morbidity and 4.67 times increased bad outcome than patients having low serum uric acid concentration.
\end{abstract}

Key words: Coronary artery disease, Serum uric acid, Mortality, Morbidity

Introduction:

Uric acid is the final oxidation product of purine catabolism in human and higher primates. In last metabolic step, the conversion of hypoxanthine to uric acid is regulated by the enzyme xanthine oxidoreductase (XO). During this process reactive oxygen species (ROS) are produced. XO (xanthine oxidoreductase) activity is up-regulated in many cardiovascular diseases, such as ischaemia to myocardium, left ventricular remodeling following myocardial infarction and heart failure. ${ }^{1}$ Uric acid play a pivotal role in the progression of atherosclerosis. Moreover, increased uric acid levels may be associated with increased platelet adhesiveness, and this effect could potentiate thrombus formation. $^{2}$

The pathophysiological link between elevated serum uric acid (SUA) and atherosclerosis are endothelial dysfunction and inflammation. ROS (reactive oxygen species) production by XO (xanthine oxidoreductase) can induce endothelial dysfunction by reducing bioavailability of nitric oxide. Endothelial dependent vasodilation is impaired in hyperuricemia patients without any overt or other cardiovascular disease. ${ }^{3}$ There is study that patients who have high uric acid level, they have more chance of short term adverse events. ${ }^{4}$ Serum uric acid levels are strong independent predictor of cardiovascular mortality in middle aged men without clinical cardiovascular disease. ${ }^{5}$ Higher serum uric acid on admission was independently associated with higher in hospital mortality. ${ }^{6}$ In hospital mortality rate is significantly higher in patients with elevated uric acid than those with low uric acid. Major adverse cardiac events (MACE) are also higher in raised uric acid. ${ }^{7}$ Hyperuricemia patients with acute myocardial infarction have a higher rate of left ventricular systolic, diastolic dysfunction and MACE.

Materials and Methodology:

This study was carried out in the department of Clinical Pathology,BSMMU Dhaka from October 2011 to September 2012. In this case series study, 108 diagnosed patients of acute myocardial infarction free from gout, renal disease, hepatic disorder and other malignant conditions were enrolled from the cardiology department of BSMMU 
and BIRDEM, Dhaka. Among 108 patients, 84 patients were male and 24 patients were female. Only patients of acute MI were enrolled in the study. All subjects were categorized into Group-1(SUA less than $6.5 \mathrm{mg} / \mathrm{dl}$ ) and Group-II(SUA more than $6.5 \mathrm{mg} / \mathrm{dl})$. All enrolled subjects were explained about the nature and purpose of the study and their informed consent were taken. Then $4 \mathrm{ml}$ blood was taken within 24 hours of attack from all study subjects. Blood was allowed to clot and centrifuged. Separated serum was collected into plastic micro centrifuged tube. Then they were stored at -20 degree centigrade until analysis. All patients were managed according to standard management protocol during their hospital stay. During hospital stay their outcome (good or bad) were recorded. Bad outcome included mortality and morbidity. Morbidity included cardiac arrhythmia, heart block, cardiogenic shock and acute left ventricular failure. Finally their outcome, good or bad (mortality and morbidity) were evaluated on the perspective of their base line serum uric acid level. Analysis was done by SPSS (Statistical package for social science) by applying appropriate formula.

\section{Result and observation}

In this case series study, 108 diagnosed patients of acute myocardial infarction were enrolled. Among 108 patients, 84 patients were male and 24 patients were female with the mean age of $54.62 \pm 12.14$ years in group-I (range 32-80) and $60.29 \pm 12.13$ years in group-II(range $35-85$ ).
Table I shows distribution of SUA in both group. The mean uric acid was found $5.43 \pm 0.72 \mathrm{mg} / \mathrm{dl}$ in group I and $8.22 \pm 1 \mathrm{mg} / \mathrm{dl}$ in group II. The mean uric acid difference was statistically significant $(\mathrm{P}<0.05)$ between two groups in unpaired t-test.

Table II shows good outcome, mortality and morbidity in both groups. Good outcome was found $43(65.2 \%)$ in group I and $13(31.0 \%)$ in group II. Mortality was $2(3.0 \%)$ in group I and 6(14.3\%) in group II. Morbidity was found $21(31.8 \%)$ and $23(54.8 \%)$ in group I and group II respectively. Good outcome, mortality and morbidity were statistically significant $(\mathrm{P}<0.05)$. This table depicts patients with raised SUA had increased mortality and morbidity than lower SUA group. On the other hand lower SUA group had better outcome than raised SUA group.

Table III shows outcome in both groups. Good outcome was high in group-I and bad outcome was high in groupII. Good outcome was found $43(65.2 \%)$ in group I and $13(31.0 \%)$ in group II. Bad outcome (mortality + morbidity) was $23(34.8 \%)$ and $29(69.0 \%)$ in group I and group II respectively. The mean outcome was statistically significant $(\mathrm{P}<0.05)$ between two groups in Chi-square test.

Table IV shows adjusted odds ratio of mortality (5.38), morbidity (4.10) and bad outcome(4.67). A subject having higher uric acid level had 5.38 (95\% CI $1.918 \%$ to $60.244 \%$

Table-I

Distribution of the study patients according to uric acid level (mg/dl) ( $n=108)$.

\begin{tabular}{lccc}
\hline & Group I(n=66) & Group II(n=42) & P value \\
& Mean \pm SD & Mean \pm SD & $0.001^{\mathrm{s}}$ \\
\hline Uric acid level (mg/dl) & $5.43 \pm 0.72$ & $8.22 \pm 1$ & $(6.7-10.5)$ \\
Range (min-max) & $(3.6-6.4)$ & & \\
\hline
\end{tabular}

ns $=$ not significant

$\mathrm{P}$ value reached from unpaired $\mathrm{t}$-test

Table-II

Distribution of the study patients according to outcome $(n=108)$.

\begin{tabular}{|c|c|c|c|c|c|}
\hline \multirow[t]{2}{*}{$\overline{\text { Outcome }}$} & \multicolumn{2}{|c|}{ Group I(n=66) } & \multicolumn{2}{|c|}{ Group II $(n=42)$} & \multirow[t]{2}{*}{$P$ value } \\
\hline & $\mathrm{n}$ & $\%$ & $\mathrm{n}$ & $\%$ & \\
\hline Good & 43 & 65.2 & 13 & 31.0 & $0.001^{\mathrm{s}}$ \\
\hline Mortality & 2 & 3.0 & 6 & 14.3 & $0.020^{\mathrm{s}}$ \\
\hline Morbidity & 21 & 31.8 & 23 & 54.8 & $0.001^{\mathrm{s}}$ \\
\hline Cardiogenic Shock & 2 & 3.0 & 6 & 14.3 & $0.037^{\mathrm{s}}$ \\
\hline Acute LVF & 8 & 12.1 & 12 & 28.6 & $0.004^{\mathrm{s}}$ \\
\hline Arrhythmia & 5 & 7.6 & 3 & 7.1 & $0.545^{\mathrm{ns}}$ \\
\hline Heart Block & 6 & 9.1 & 2 & 4.8 & $0.626^{\mathrm{ns}}$ \\
\hline
\end{tabular}

$\mathrm{s}=$ significant, $\mathrm{ns}=$ not significant

$P$ value reached from chi square test. 
Table-III

Distribution of the study patients according to outcome $(n=108)$.

\begin{tabular}{lccccccc}
\hline Outcome & \multicolumn{2}{c}{ Group I(n=66) } & & \multicolumn{2}{c}{ Group II(n=42) } & Total $(n=108)$ & $P$ value \\
\cline { 2 - 3 } & $\mathrm{n}$ & $\%$ & & & $\%$ & & \\
\hline Good & 43 & 65.2 & & 13 & 31.0 & 56 & $0.001^{\mathrm{s}}$ \\
Bad & 23 & 34.8 & & 29 & 69.0 & 52 & \\
\hline
\end{tabular}

$\mathrm{s}=$ significant

$\mathrm{P}$ value reached from chi square test.

Table-IV

Logistic regression considering uric acid level as independent variables and outcome as dependent variable $(n=108)$.

\begin{tabular}{lcccc}
\hline & Adjusted & \multicolumn{2}{c}{$95 \%$ CI } & P \\
\cline { 2 - 4 } & OR & Lower & Upper & Value \\
\hline Mortality & 5.38 & 1.918 & 60.244 & $0.007^{\mathrm{s}}$ \\
Morbidity & 4.10 & 1.720 & 9.750 & $0.001^{\mathrm{s}}$ \\
Bad outcome & 4.67 & 2.019 & 10.820 & $0.001^{\mathrm{s}}$ \\
Constant & 0.28 & - & - & $0.001^{\mathrm{s}}$ \\
\hline
\end{tabular}

$\mathrm{p}<0.007)$ time increased mortality. A subject having higher uric acid level had $4.10(95 \%$ CI $1.720 \%$ to $9.750 \%$ p $<0.001)$ time increased morbidity. A subject having higher uric acid level had $4.67(95 \%$ CI $2.019 \%$ to $10.820 \% \mathrm{p}<0.001)$ time increased bad outcome.

\section{Discussion:}

Hyperuricemia was postulated to be a risk factor for coronary artery disease (CAD) more than 5 decades ago. In recent decades, serum uric acid has been recognized as a potential risk factor for $\mathrm{CVD}^{8}$. Others reported that it was only confounded by the relation of uric acid with conventional risk factors for Coronary artery disease. ${ }^{9}$ Serum uric acid level had a significant statistical relation with cardiovascular disease or mortality due to cardiovascular disease. ${ }^{10}$

The result of present study revealed, mortality was $2(3.0 \%)$ in group I and 6(14.3\%) in group II. Mortality was statistically significant $(\mathrm{P}<0.05)$. On the other hand lower SUA group had better outcome than raised SUA group. Lazzeri et al. ${ }^{11}$ found that In-hospital mortality was higher in "high" SUA patients ( $9.0 \%$ vs. $2.5 \%)$, p $<0.006$. Kojima $S$ et al(2005) made a comparison of mortality between $4^{\text {th }}$ quartile and $1^{\text {st }}$ quartile of uric acid which was $11 \%$ vs $2 \%$ and our study revealed it was $14.3 \%$ vs $3 \%$ in group-II and group-I. Bickel $\mathrm{C}$ et al. ${ }^{12}$ made a comparison between highest quartile of uric acid than lowest quartile of uric acid. The mortality rate was increased from $3.4 \%$ to $17.1 \%$ (five fold increase). These findings are consistent with our study.

The present study revealed that morbidity was $21(31.8 \%)$ and $23(54.8 \%)$ in group I and group II respectively. Morbidity were statistically significant $(\mathrm{P}<0.05)$. Bae J H et al. ${ }^{13}$ evaluated the effects of the serum uric acid levels on major adverse cardiovascular events (MACEs). MACE rate increased from $7.2 \%$ to $20.1 \%$ (Lowest quartile vs highest quartile). This is consistent with our study. Hyan DW et al. ${ }^{14}$ showed that for all patients highest uric acid quartile was associated with increased risk of MACE $(\mathrm{p}=0.000)$. Basher $\mathrm{N}$ et al. ${ }^{15}$ showed that mortality was $2.8 \%$ in lower uric acid group and $6.6 \%$ in higher uric acid group $(\mathrm{p}<0.01)$. MACE also increased from $5.7 \%$ to $11.1 \%$ from non-hyperuricemia to hyperuricemia $(\mathrm{p}<0.01)$. These findings are consistent with our study.

In our study incidence of acute LVF was $28.6 \%$ and $12.1 \%$ in case of raised SUA group and low SUA group. Chen Li et al. ${ }^{16}$ demonstrated that left ventricular dysfunction was $36.4 \%$ vs $15.1 \%,(p<0.001)$ in case of hyperuricemia and non- hyperuricemia. Our study revealed consistent findings.

Our study revealed good outcome was high in group-I and bad outcome was high in group-II. Good outcome was found $43(65.2 \%)$ in group I and $13(31.0 \%)$ in group II. Bad outcome (mortality + morbidity) was 23(34.8\%) and $29(69.0 \%)$ in group I and group II respectively. The mean 
outcome was statistically significant $(\mathrm{P}<0.05)$ between two groups in Chi-square test.

In the present study we have found adjusted odds ratio for mortality was 5.38(95\% CI, 1.918-60.244, $<<0.007)$ and morbidity was $4.10(95 \% \mathrm{CI}, 1.720-9.750, \mathrm{p}>0.001)$. This indicates a patient having higher uric acid level had 5.38 times increased mortality and 4.10 times increased morbidity than patients with low uric acid level. Kaya MG et al (2012) revealed that high plasma UA levels were an independent predictor of major adverse cardiac events in the hospital (odds ratio 2.03, 95\% confidence interval 1.25 to3.75, $\mathrm{p}=0.006)$. MACEs were more frequent in hyperuricemia (16\% vs 7\%) and in hospital mortality rate was higher in patients with high UA levels ( $9 \%$ vs $2 \%$, p $<0.001)$. Valente S et al. ${ }^{17}$ had a study on STEMI patients presenting with a cardiogenic shock. On admission SUA $>6.5 \mathrm{mg} / \mathrm{dl}$ was associated with higher odds of in-hospital death, OR=6.7 (95\% CI 1.4-31.8). Kojima et al(2005) demonstrated that mortality was 3.7 times higher in highest quartile of uric acid than lowest quartile of uric acid(OR=3.7 $95 \% \mathrm{CI}, \mathrm{p}<0.0004)$. Lazzeri et al(2010) found that risk of mortality was 3.9 times more in patients with hyperuricemia than normal uric acid(OR,3.9, 95\% CI, p<0.01). These findings are consistent with our study.

\section{Conclusion:}

On admission serum uric acid estimation is a good predictor of in hospital bad outcome in patients with acute myocardial infarction. As it is a cheap and non invasive procedure, it can be routinely practiced in cardiac emergency department for risk stratification of patients. This can guide the physicians which patients will be monitored closely.

\section{References:}

1. Iliesiu A, Campeanu A, Dusceac D, 'Serum uric acid and cardiovascular disease', Maedica-A journal of clinical Medicine, 2010;5(3):186-192.

2. Weir JC, Muir S, Walters M, Lees KR, 'Serum urate as an independent predictor of poor outcome and future vascular events after acute stroke', Stroke, 2003; 34:1951-1957.

3. Kato M, Hisatome I, Tomkuru Y, Kotani K, Kinugawa T, Ogino K, Ishida K et al., 'Status of endothelial dependent vasodilatation in patients with hyperuricaemia', American njournal of cardiology, 2005;96:1576-1578.

4. Kojima S, Sakamoto T, Ishihara M, Kimura K, Miyazaki $\mathrm{S}$, Yamagishi $\mathrm{M}$ et al., 'Prognostic usefulness of serum uric acid after myocardial infarction(The Japanese Acute Coronary Syndrome Study)', Am J Cardiol, 2005;96:489-495.

5. Niskanen LK, Laaksonen DE, Nyyssonen K, Alfthan G, Lakka HM, Lakka TA, Salonen JT, 'Uric acid level as a risk factor for cardiovascular and all-cause mortality in middle-aged Men', Arch. Intern. Med, 2004; 164:1546-1551.

6. Car S, Trkulja V, 'Higher serum uric acid on admission is associated with higher short term mortality and poorer longterm survival after Acute Myocardial Infarction : Retrospective Prognostic study', CMJ, 2009; 50:559-566.

7. Kaya MG, Uyarel H, Akpek M, Kalay N, Ergelen M, Ayhan E, et al., 'Prognostic value of uric acid in patients with STElevated myocardial infarction undergoing primary coronary intervention', The American Journal of Cardiology, 2012; 109:486-491.

8. Chen JH, Chuang SY, Chen HJ, Yeh WT, Pan WH, 2009, 'Serum Uric Acid Level as an Independent Risk Factor for AllCause, Cardiovascular, and Ischemic Stroke Mortality: A Chinese Cohort Study', Arthritis \& Rheumatism, vol. 61, No.2; pp 225-232.

9. Goodarzynejad H, Anvari MS, Boroumad MA, Karimi A, Abbasi SH, Davoodi G, 'Hyeruricaemia and the presence and severity of coronary Artery Disease', Lab Medicine, 2010; 41(1):40-45.

10. Nozari Y and Geraiely B, 'Corelation between the serum level of uric acid and HS-CRP with the occurrence of Early Systolic Failure of left ventricle following Acute Myocardial infarction', Acta Medica Irania, 2011; 49(8) :531-535.

11. Lazzeri C, Valente S, Chiostri M, Sori A, Bernardo P, Gensini $\mathrm{G} F$ et al., 'Uric acid in acute phase of ST elevation myocardial infarction submitted to primary PCI: Its prognostic role and relation with inflammatory markers A single centre experience', International Journal of Cardiology, 2010;138:206-216.

12. Bickel C, Rupprecht H, Blankenberg S, Rippin G, Hafner G, Daunhauer A, 'Serum Uric Acid as an Independent Predictor of Mortality in Patients With Angiographically Proven Coronary Artery Disease', Am J Cardiol,2002; 89:12-17.

13. Bae JH, 'Serum Uric Acid is Associated with Cardiovascular Events in Patients with Coronary Artery Disease', Korean Circulation J, 2007; 37:161-166.

14. Hyun DW, Kim KH, Yoon HJ, Kwon TG, Kim KY, Bae JH et al., "Gender difference in the role of serum uric acid for predicting cardiovascular events in patients with coronary artery disease', Korean Circulation J, 2007; 37:196-201.

15. Basar N, San N, Ozcan F, Erden G, Kanat S, Sokmen E, Isleyen A et al., 'Elevated Serum Uric Acid Predicts Angiographic Impaired Reperfusion and 1-Year Mortality in ST-Segment Elevation Myocardial Infarction Patients Undergoing Percutaneous Coronary Intervention', J Investig. Med, 2011; 59:931-937.

16. Chen Li, Li X, Qiao W, Ying Z, Qin Y, Wang Y, Zeng Y, Ke $\mathrm{Y}$, 'Serum uric acid in patients with acute ST-elevation myocardial infarction', World Journal of Emergency Medicine, 2012; 3(1):35-39.

17. Valente S, Lazzeri C, Vecchio S, Giglioli C, Margheri M, Bernardo $\mathrm{P}$ et al. 'Predictors of in-hospital mortality after percuteneous coronary intervention for cardiogenic shock', International Journal of Cardiology, 2007; 114:176-182. 